\title{
Detecting the gravito-magnetic field of the dark halo of the Milky Way - the LaDaHaD mission concept
}

\section{LaDaHaD}

\author{
Angelo Tartaglia ${ }^{1,2}\left[\right.$ (D) Massimo Bassan ${ }^{3} \cdot$ Lorenzo Casalino $^{4}$. \\ Mariateresa Crosta ${ }^{2}$. Mario Lattanzi ${ }^{2}$. Enrico Lorenzini ${ }^{5}$. \\ David Lucchesi ${ }^{6}$. Roberto Peron ${ }^{6}$. Giuseppe Pucacco ${ }^{3}$. \\ Matteo Luca Ruggiero ${ }^{7}$. Francesco Santoli ${ }^{6}$ Pavol Valko ${ }^{8}$. \\ Alberto Vecchiato $^{2} \cdot$ Francesco Vespe $^{9} \cdot$ Massimo Visco $^{6}$
}

Received: 2 September 2020 / Accepted: 27 January 2021 / Published online: 9 March 2021 (C) The Author(s) 2021

\begin{abstract}
We propose to locate transponders and atomic clocks in at least three of the Lagrange points of the Sun-Earth pair, with the aim of exploiting the time of flight asymmetry between electromagnetic signals travelling in opposite directions along polygonal loops having the Lagrange points at their vertices. The asymmetry is due to the presence of a gravito-magnetic field partly caused by the angular momentum of the Sun, partly originating from the angular momentum of the galactic dark halo in which the Milky Way is embedded. We list also various opportunities which could be associated with the main objective of this Lagrange Dark Halo Detector (LaDaHaD).
\end{abstract}

Keywords Gravito-magnetism · Dark halo · Lagrange points ·

Solar gravito-magnetism $\cdot$ Relativistic Positioning

Angelo Tartaglia

angelo.tartaglia@inaf.it

1 Politecnico di Torino, DISAT, Corso Duca degli Abruzzi 24, 10129, Torino, Italy

2 OATo, INAF, Pino Torinese, Italy

3 Dipartimento di Fisica, INFN and Università di Roma Tor Vergata, Roma, Italy

4 Politecnico di Torino-DIMEAS, Torino, Italy

5 DII, University of Padova, Padua, Italy

6 IAPS, INAF, Roma, Italy

7 LNL, INFN, Legnaro, Italy

8 Physics Department, Slovak University of Technology in Bratislava, Bratislava, Slovakia

9 Centro di Geodesia Spaziale - Matera, ASI, Matera, Italy 


\section{Introduction}

The present paper proposes future mission(s) in the inner Solar System aimed at detecting weak gravitational effects of General Relativity (GR) especially in connection with the possible existence of a galactic dark halo. The main configuration is designed for an experiment which per se requires more than one spacecraft but allows for a progressive deployment and various steps. Each step can provide essential information; furthermore, synergies are possible with other programs. For short we shall nickname our proposal LaDaHaD (Lagrange Dark Halo Detector). The main scientific objective is to look for evidence of gravito-magnetic (GM) effects due to the angular momentum of the Milky Way, both in its visible and in its dark component. As it is well known, General Relativity implies that any rotating mass distribution gives rise to a peculiar gravitational interaction resembling the effect of a magnetic field on moving charges. If the Milky Way (MW), as it is now a standard assumption, is immersed in a dark halo much more massive than the visible stars of the galaxy, it is reasonable to look for any effect originating from the rotation of such a huge halo. We expand the goal of the proposed mission(s) to include any detectable gravito-magnetic effect over distances of the order of the inner Solar System. In addition to the aforementioned angular momentum of the Milky Way, we can include the detection and measurement of the solar gravito-magnetic field. So far GM fields have been looked for at the local scale in the terrestrial environment [12, 23, 49], and at an astronomical scale, in order to verify the compatibility between their presence and observations of the behavior of binary systems comprised of very compact objects such as neutron stars [31]. The mission(s) are aimed at sensitivities and accuracies better than the results obtained so far for the terrestrial effect. We note that the experiment could also verify or strongly constrain deviations from GR, especially the ones expressed by the PPN parameters $\alpha_{1}$ and $\gamma$.

The envisaged experimental setup consists in placing spacecraft in the Lagrange points of the Sun-Earth pair. The task assigned to each station is to bounce electromagnetic signals (pulses) along closed paths between the Lagrange points and the Earth. The advantage of the Lagrange points system is that it is tied to the Earth and rotating with it; furthermore, all stations are in free fall and locally equivalent to inertial laboratories.

The essence of the experiment would be the local measurement of times of flight along the closed paths, made at one of the stations (or the Earth). More specifically the data of interest would be the time of flight differences between clockwise and counterclockwise beams along the same path, which are directly connected with the presence of a gravito-magnetic field and the kinematical rotation of the whole apparatus.

The envisaged setup would also allow for a zero-area Sagnac interferometer configuration [45] at the scale of millions of kilometers, which would allow monitoring of Gravitational Waves (GW).

The same stations at the Lagrange points could act as artificial pulsars and bases of a Relativistic Positioning System usable for the guidance of space missions in the Solar System. 
The main technological and methodological challenges to be tackled concern the reliable measurement of time intervals as short as $10^{-17} \mathrm{~s}$ or less, in a space environment. The whole communication and signal management system also needs to be properly designed and materially implemented. Since the stations cannot be rigidly fixed at the Lagrange points, it is also necessary to properly reconstruct, then control, their movements around each point.

Last but not least, sending missions to $L_{4}$ and $L_{5}$ offers the opportunity to explore the local environment of those shallow effective potential wells, which are not well studied.

The complete experimental setup could be deployed gradually, starting with the Earth and already planned (for different purposes) or even flying missions, plus one station in $L_{5}$. The corresponding triangle would already permit us to start gathering data; then the other stations could be launched one by one, progressively enriching and upgrading the system.

\section{Background of the proposal}

General Relativity is perhaps the most elegant and important theory of contemporary physics for describing the behavior of the Universe at the largest scale. Despite being so successful, there are a number of major open problems: the most fundamental and difficult-to-reconcile one is the deep incompatibility between GR and quantum mechanics; then we have the dark matter (DM) mystery; third in the list, but no less in important, is the accelerated expansion of the Universe or dark energy (DE) problem. Indeed, we know that, according to the standard cosmological $\Lambda C D M$ model, the dominant form of energy-mass density in the Universe exists as nonrelativistic (cold) dark matter and negative pressure dark energy, while ordinary (baryonic) matter, forming all visible stars and interstellar (intergalactic) dust, contributes less than 5\% [38]. Evidence for presence of DM is known at several levels [24] and a substantial amount of DM is required to explain the stability of galaxies [42], the permanence of clusters of galaxies, gravitational lensing [36], and the spectrum of cosmic microwave back-ground radiation. The physical nature of DM is intensively discussed and searched for. Early investigations concentrated on ordinary matter in invisible (nonluminous) objects; nowadays, massive particles interacting only very weekly with ordinary matter (WIMPs) and/or very light axions are leading candidates. None of the recent experiments, however, has been able to detect them.

Spiral galaxies, for which rotational curves measurement could be extended into stars-free edge regions via radio emission observations of neutral hydrogen [43], are the best test beds for assessing DM scenarios. When observing the peripheral tangential (rotation) motion of the stars (and of neutral hydrogen atoms) in such galaxies, one concludes that the centripetal attraction due to the visible mass is not strong enough to explain the measured velocities. For long term stability of the galaxy some kind of additional centripetal force is necessary. The mutual gravitational attraction of stars, central black holes and interstellar dust provide a significant part (nearly all) of the required centripetal force at small distances (up to $5 \mathrm{kpc}$ ) from the centre, while flat rotation curves at larger distances undeniably point towards some other 
source of centripetal force. In the DM scenario this extra force is considered to be the gravitational attraction of a large halo (far beyond $30 \mathrm{kpc}$ in diameter) of massive nonluminous particles with an isothermal spherical distribution.

The stabilizing effect of DM is currently being contemplated only as a consequence of static or slowly changing gravitational fields (the $g_{00}$ component of the metric tensor). However, the presence of dynamical components $\left(g_{0 i}\right)$, also known as gravito-magnetic components, might successfully mimic, or at least contribute to, the effects of DM in rotating spirals. However, it is true that the contribution of such dynamical fields would be too weak if it were only due to the angular momentum of ordinary matter in galaxies. We can estimate the GM effect of the rotating visible matter in disc-dominated spiral galaxies assuming that the surface brightness corresponds to ordinary (baryon) matter density in the disc. An oversimplified model treats the system as an infinitely thin disc with an exponentially decreasing mass distribution

$$
\sigma(r)=\Sigma_{0} e^{-r / r_{d}}
$$

where $r_{d}$ is a characteristic disc scale length and $\Sigma_{0}$ the surface mass density parameter. Under these conditions and for a $220 \mathrm{~km} / \mathrm{s}$ peripheral velocity of the plateau of the rotation curve (which is the case of the MW), the intensity of the GM field due to the visible mass would be as shown in Fig. 1.

Calculated gravito-magnetic field strengths, shown in Fig. 1, are very weak, varying from positive values of $\sim+2.4 \times 10^{-21} \mathrm{~s}^{-1}$ at bulge edge, to $\sim-1.5 \times 10^{-22} \mathrm{~s}^{-1}$ at the minimum, while crossing zero, then changing sign, at approximately $2 r_{d}$. This

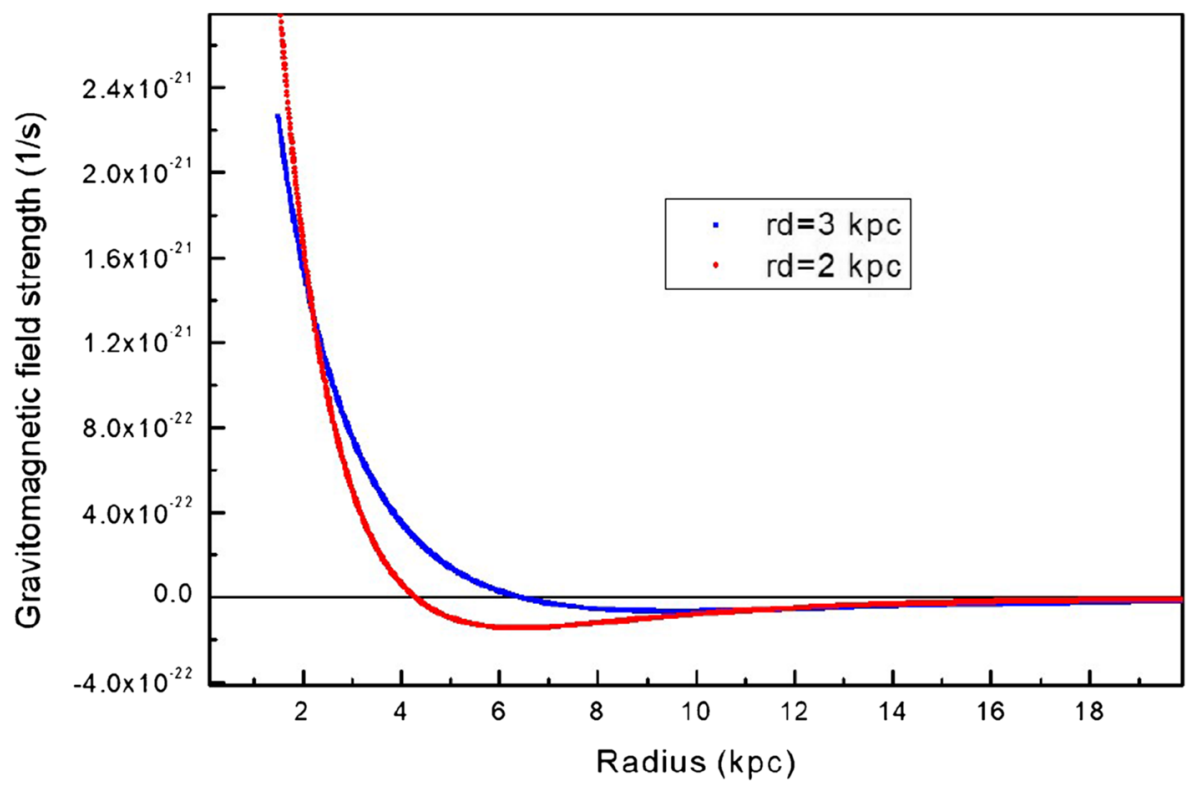

Fig. 1 Calculated GM field strength generated by ordinary matter of orbiting stars situated in an infinitely thin galaxy disc, with mass distribution given by (1), for a visible disc mass $4.5 \times 10^{10}$ solar masses and constant $220 \mathrm{~km} / \mathrm{s}$ plateau orbital velocity. The possible central black hole and bulge contribution are not accounted for. [Valko, P.: courtesy of the author (2019)] 
behaviour is clearly related to the assumed (observed) exponential ordinary mass distribution given by (1). Such feeble gravito-magnetic fields are weaker than similar fields in the vicinity of typical rotating stars. For instance, the Sun generated GM field strength at Earth's distance is $\sim 8.3 \times 10^{-20} \mathrm{~s}^{-1}$. Such fields are too weak to be sensed by any current experimental detection scheme.

The rotational velocity terms associated with the DM scenario for M31 [7] provide a linearly growing contribution to the rotation curve with a slope of $\sim 1.2 \times 10^{-16}$ $\mathrm{s}^{-1}$. This result could be interpreted also as the influence of a homogenous GM field, perpendicular to the plane of the disc of the galaxy, with an intensity $B_{G M} \sim$ $1.2 \times 10^{-16} \mathrm{~s}^{-1}$.

Considering our Galaxy, if the Sun should move around the centre of the Milky Way solely under the effect of a uniform GM field, ignoring for a moment purely attractive gravitational forces of both ordinary and dark matter, the field strength would have to be $B_{G M} \sim 4.5 \times 10^{-16} \mathrm{~s}^{-1}$. On the other hand, according to a realistic MW mass distribution model [25], an additional force component is needed, to account for $30 \mathrm{~km} / \mathrm{s}$ of orbital velocity from the total $v_{L S R}=220 \mathrm{~km} / \mathrm{s}$. The Local Standard of Rest velocity, $v_{L S R}$, is the average tangential velocity of matter in the neighbourhood of the Sun with respect to the centre of the MW. A local value of $B_{G M} \sim 1.1 \times 10^{-16} \mathrm{~s}^{-1}$ would account for this additional centripetal force.

In fact, exploiting the analogy with classical electromagnetism, the presence of a GM field would produce on moving masses a force similar to the EM generalized Lorentz force. The induced three-acceleration $\bar{a}$ can be written as [39]:

$$
\bar{a} \cong-c^{2} \bar{\nabla} g_{00}+2 \bar{v} \times \bar{B}_{G M}
$$

where the gradient of $g_{00}$ (time-time component of the metric tensor) is in practice the gradient of the Newtonian gravitational potential and $\bar{v}$ is the velocity of the test mass.

These fascinating enigmas and the above-mentioned intriguing possibilities drive the interest to foster experimental (and theoretical) research aimed at verifying all implications of GR, both in the extremely high and in the ultralow energy domains. At the scale of the Solar System, all GR effects lie in the weak and ultra-weak gravity range. Much of the interest of science is concentrated on the confirmation of, and possible deviations from, the behavior predicted by the theory. The scope of the present proposal is to envisage the opportunity of an experimental setup and measurement technique able to provide evidence for the presence of GM effects associated with the rotation of the dark matter halo of the Milky Way (if it exists); additional information could be retrieved from such an experiment: from possible deviations from GR to the measurement of the angular momentum of the Sun, and more.

\section{Experimental rationale}

Among the facts worth investigation, as we have seen, are the effects of the rotation of the source of gravity. According to GR, a mass does not simply produce attraction towards other masses, but generates also an additional interaction, dragging test bodies around with the proper rotation of the source and giving rise to a force according 
to (2). This contribution to the gravitational interaction is known as gravito-magnetic field or, at least in its most well-known form, as the Lense-Thirring (LT) effect [33, 53]. Direct measurements of LT have been performed in the space surrounding Earth with our planet as the source. The effect has been verified analyzing the precession of four gyroscopes carried by a satellite in a polar orbit (GP-B experiment) with a 19\% accuracy [23]. A better result (10\% accuracy) has been obtained studying the orbits of the LAGEOS and LAGEOS2 satellites, reconstructed by means of laser ranging techniques, $[10,11]$. The result has been improved, reaching a 5\% accuracy, in the LARES experiment (still under way) [12]. After a refinement of the analysis of the usual (Newtonian) field of the Earth and of the non-gravitational perturbations of the orbit, performed by the LARASE Team, the evaluation of the accuracy has now been brought to $2 \%$ [35].

All these tests need averaging or integrating over values of the GM field of the Earth along the orbits of the probes; furthermore, in order to disentangle the proper LT effect from the ordinary gravitational attraction, a detailed modeling of the latter around the planet is required. A different approach is envisaged by the GINGER experiment, now at the R\&D stage [4]. In this case the measurement would be local and performed at a fixed position on (or near) the surface of the Earth [6, 21, 49]. Besides these experiments, indirect evidence for GM effects is found in the dynamics of binary star systems and in particular in the spin-orbit coupling for the companion stars [55]. The idea we propose here is inspired by the same principle on which the ring lasers of GINGER work. If we force light to move along a path closed in space (by means of optical fibers or mirrors) and let the whole apparatus rotate, the righthanded and left-handed beams in it take different times to return to the emission point. This is the essence of the classical Sagnac effect [41]; the effect, contrary to what Georges Sagnac thought, is a relativistic one. When a gravitational field is present, besides the purely kinematic effect of rotation, additional GR terms appear in the form of effective rotations and accounting for both the coupling between rotation of the device and the ordinary gravitational attraction, and the GM field (LT drag).

Our proposal is to make an experiment in space, working at the scale of the internal Solar System, but based on local time measurements: the source of the gravitational effects could be the angular momentum of the Milky Way, both of its visible and its dark component, and/or of any other possible source of GM field, including a possible primordial relic field (similar to the magnetic relic field [44]). We expect the dark halo to rotate since the ordinary matter in the galaxy rotates. Usually the galactic dark halos are assumed to be non-rotating. The assumption has practical motivations, but would correspond to a very peculiar situation in the Universe: non-rotation, if taken literally, is an absolute condition (like, locally, for spin 0 particles). However, the conjecture of co-rotation of the halo with the embedded galaxy seems reasonable. The mutual gravitational interaction between baryonic and non-baryonic matter, considering that the galactic spirals are not homogeneous, must produce a reciprocal drag and the apparent stability of the configuration of the Milky Way makes the equal rotation rates of the two components credible. The problem has been considered when referred to the rotation of gas halos of galaxies [26, 46] and the analogy looks reasonable. If so, the mass commonly attributed to the dark halo suggests that the associated GM effect would not be entirely negligible with respect to the usual 
direct attraction (gravito-electric effect). Besides the aforementioned possible galactic GM, another source which will in any case be present is the angular momentum of the Sun: on one side it would be interesting to measure it, in order to retrieve independent information on the internal structure of our star; on the other, a problem to solve will be how to discriminate the solar effect from the galactic contribution.

Instead of measuring a beat frequency produced by the superposition of two stationary counter-rotating electromagnetic beams, as in the case of ring lasers, the idea is now to directly measure the time of flight asymmetry between two electromagnetic pulses travelling along the same space trajectory in opposite directions. In order for this idea to be implemented we need: a) a long enough path to produce a detectable time of flight difference; and b) a stable, possibly non-rotating device (in the reference frame of the observer) in order to remove purely kinematic effects. A stable configuration is found in space when considering the Lagrange points $\left(L_{i}\right)$ of a two body gravitationally bound system. A remarkable property of the $L$ points is that they keep (almost) fixed positions with respect to the rotating pair of masses. There are five $L$ points for any pair of objects, where the centrifugal force is balanced by the gravitational attraction towards the barycentre of the pair. Let us consider the SunEarth pair: a spacecraft located in one of the L points will orbit the centre of mass of the system (which lies within the body of the Sun) at the same angular velocity as the Earth does, so keeping the relative position fixed. This would literally be the case only if the orbit of the Earth were perfectly circular, which it is not; however, the effect of the eccentricity, as well as other perturbations and deviations are, in principle, manageable. The properties of the Lagrange points have already been, and continue to be, exploited for science purposes, especially using $L_{2}$ (lying along the Sun-Earth line on the same side, but outwardly with respect to the Earth), and $L_{1}$ (as $L_{2}$, but internal to the Sun-Earth pair). We can benefit from the Gaia mission, positioned in $L_{2}$, through the exploitation of the fully relativistic framework implemented for the data analysis of that mission in the ever-present and ever-changing Solar System overlapping gravitational fields.

\subsection{The measurement strategy}

Consider a spacecraft placed at any of the L points, and repeaters (transponders) in the others. The situation is sketched in Fig. 2.

From the station, a couple of electromagnetic pulses are sent towards two other L points; the transponders there re-send the pulses to each other, then back to the main station. $L_{3}$ is more problematic since it is situated behind the Sun and not visible from Earth; it would undoubtedly be more difficult to put a spacecraft there and to exchange data with it. However, $L_{3}$ is visible from $L_{4}$ and $L_{5}$, which could work as relay stations, and it could offer an interesting and advantageous configuration for the measurement we are proposing. Setting $L_{3}$ aside for a moment, a possible choice could correspond to the sequence $L_{2}-L_{4}-L_{5}-L_{2}$ and viceversa. As mentioned before, the times of flight in opposite directions would be different. Indeed, writing the line element for a space-time having a chiral symmetry about the time axis (i.e. 
Fig. 2 Positions of the Lagrange points. The yellow disc is the Sun; the smaller blue disc is the Earth

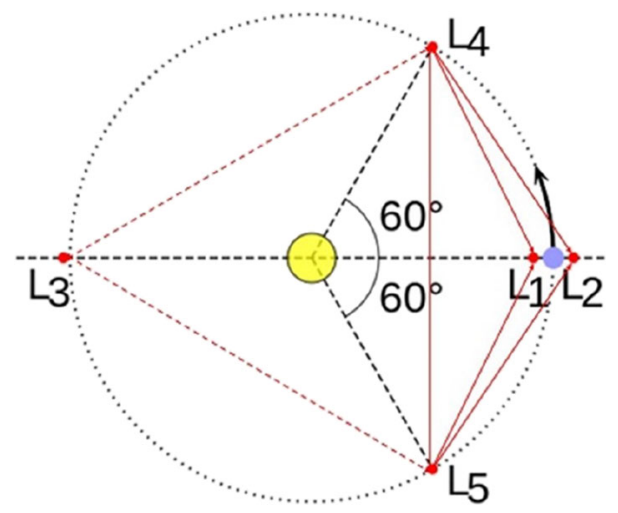

having a gravito-magnetic field) and with an axially symmetric central mass, one has (general coordinates)

$$
d s^{2}=g_{00} c^{2} d t^{2}+g_{i j} d x^{i} d x^{j}+2 c g_{0 i} d x^{i} d t
$$

The mixed term $g_{0 i}$, in the weak field approximation leading to the GM field, may be interpreted as a space component of a potential three-vector $\bar{h}$ similar to the vector potential of classical electromagnetism. Fully exploiting the analogy, the GM field is then:

$$
\bar{B}_{G M}=c \bar{\nabla} \times \bar{h}
$$

For electromagnetic signals $d s=0$, so from (3) we may obtain the coordinated time of flight element $d t$. Considering a closed path, choosing a propagation toward the future, remarking that for opposite directions propagation $d x^{i}$ changes sign, and taking into account the gravitational field at the emission/arrival station, the right/left proper time of flight difference for the observer, $\delta \tau$, will be [21]:

$$
\delta \tau=-\frac{2}{c} \sqrt{g_{00}} \oint \frac{g_{0 i}}{g_{00}} d x^{i}
$$

In the case of a circuit including our L points, it is convenient to use heliocentric ecliptic space coordinates, where, in our case, $\theta=0$ (the L points lie in the ecliptic plane). In this reference frame, considering the symmetry, $g_{0 i} \equiv g_{0 \phi}$, and the corresponding elementary displacement is $d x^{i} \equiv r d \phi$.

\section{The galactic dark halo}

Evaluating the effect of the GM field of the Milky Way, we note that our Lagrangian triangle (or any other Lagrangian polygon we may consider) will be orbiting the Sun together with the Earth, hence, during the year, it will change its position with respect to the centre of the Milky Way. This periodic motion implies the "device" will encounter a changing galactic GM field, $\bar{B}_{G M}$. The maximum size of the displacement is of the order of the diameter of the Earth's orbit, i.e. $\delta l \sim 3 \times 10^{11} \mathrm{~m}$, and the distance of the Sun from the Galactic Centre is roughly $l \sim 2.5 \times 10^{20} \mathrm{~m}$ [27]. 
It is reasonable to expect that the relative change of the intensity of $\bar{B}_{G M}$ (seasonal variation) be [51]

$$
\frac{\delta B_{G M}}{B_{G M}} \sim \frac{\delta l}{l} \sim 10^{-9}
$$

If we are interested in detecting the possible GM field of our galaxy with accuracies of the order of, say, $1 \%$ we conclude that $\bar{B}_{G M}$ is practically constant over the interior Solar System. Of course $\bar{B}_{G M}$ is a vector, but we may easily extend the above conclusion to its direction also: the GM field of an axially symmetric space-time is expected to have a dipolar structure [6], so the field is expected to be perpendicular to the galactic plane all over that plane. The Sun is thought not to lie exactly on the Galactic Plane, furthermore the plane of the ecliptic is at an angle $\alpha \sim 60^{\circ}$ with respect to the Galactic Plane. Summing up, and staying within the borders of reasonable approximations, we may assume that at any time of the year, the GM field of the Milky Way is a constant vector at an angle $\alpha$ with the ecliptic north pole. This situation simplifies the evaluation of the possibly detectable signal in LaDaHaD. Recalling (4) we may use Stokes ' theorem and write:

$$
\delta \tau_{G M}=\frac{2}{c^{2}} \int \bar{B}_{G M} \cdot \hat{u}_{n} d S
$$

The flux integral is over the area, $S_{L}$, contoured by the Lagrangian triangle (or other polygon). The practical local uniformity of $\bar{B}_{G M}$ immediately gives the result of the integral:

$$
\delta \tau_{G M} \cong \frac{2}{c^{2}} B_{G M} S_{L} \cos \alpha
$$

We may reverse (8) to get $B_{G M}$ :

$$
B_{G M} \cong \frac{c^{2} \delta \tau_{G M}}{2 S_{L} \cos \alpha}
$$

The area of the $L_{4}-L_{5}-L_{2}$ triangle $^{1}$ is $\sim 9.9 \times 10^{21} \mathrm{~m}^{2}$. Let us suppose the smallest detectable time of flight asymmetry be $\sim 10^{-16} \mathrm{~s}$. In such conditions we should be able to sense GM fields of the Milky Way at the position of the Solar System:

$$
B_{G M} \geq \sim 3 \times 10^{-22} \quad s^{-1}
$$

Of course, what we would measure with $\mathrm{LaDaHaD}$ would indeed combine the effect of the visible matter and of the dark halo. To estimate the relative importance of the two contributions, let us recall that GM fields originating from rotations are in general proportional to the angular momentum of the source. A rough estimate can then be obtained comparing the angular momentum of a homogeneous disc (symbolizing baryonic matter) with that of a homogeneous sphere (the dark halo) rigidly rotating at the same angular speed. The ratio of the latter to the former is just, modulo a factor of order 1, the ratio between the two masses, which, in the case of the Milky Way could be of the order of 10. In other words, the contribution of the dark

\footnotetext{
${ }^{1}$ The triangle is treated as being Euclidean and the curvature of light rays due to the solar lensing is much smaller than the effects we are interested in.
} 
halo would clearly prevail over the contribution of ordinary matter (which has been shown in Fig. 1).

\section{Solar gravito-magnetism}

Considering the only GM field of the Sun (neglecting the small perturbation from the Earth) and recalling (3) for an axially symmetric line element in vacuo and weak field approximation, gives:

$$
g_{0 \phi}=2 \frac{G J}{c^{3} r^{2}}
$$

$J$ is the angular momentum of the Sun. In the reference frame of the observer (main station) rotating with the Earth and with the whole set of L points, introducing the astronomical unit $\rho$ (the Sun-Earth average distance $\sim 1.5 \times 10^{11} \mathrm{~m}$ ), assuming weak field conditions and keeping terms up to the lowest order in the angular momentum, (11) becomes:

$$
g_{0 \phi_{L a D a H a D}}=-\sqrt{\frac{G M}{c^{2} \rho}}\left[\frac{\rho}{r}+\left(1-2 \frac{\rho}{r}\right) \frac{G M}{c^{2} r}\right]+2 \frac{G J}{c^{3} r^{2}}
$$

with $M$ the mass of the Sun.

Let us now introduce numbers. The distance of $L_{4}$ and $L_{5}$ from the Earth (one ahead and the other behind, along the terrestrial orbit) is $\rho$. The distance of $L_{1}$ and $L_{2}$ (inward and outward along the Sun-Earth line) is $\sim 1.5 \times 10^{9} \mathrm{~m}$. Using (5), with $g_{00}=\sqrt{\left(1-2 \frac{G M}{c^{2} \rho}\right)}$, in the case of an $L_{2}-L_{4}-L_{5}-L_{2}$ circuit, we see that the total time of flight turns out to be $\sim 2000$ seconds and the right/left asymmetry is [50]

$$
\delta \tau \approx 4 \times 10^{-13} s .
$$

If one succeeded in including $L_{3}$, the triangle $L_{3}-L_{4}-L_{5}$ would correspond to a time of flight of the order of $8000 \mathrm{~s}$ and it would be $\delta \tau \geq \sim 10^{-12} \mathrm{~s}$. As an aside: if the galactic GM field able to account for the excess $v_{L S R}$ actually existed, the time of flight difference would be of the order of $\sim 10^{-11} \mathrm{~s}\left(>10^{-10} \mathrm{~s}\right.$, if $L_{3}$ were used), taking account of the angle between the ecliptic and the MW disc planes.

If the spacecraft located in $L_{2}$ (or any other point) is equipped with an atomic clock activated by the arrival of the first pulse and stopped by the arrival of the second, the above time difference may be measured at the $1 \%$ level (or better). In this way a test of the GR effects in the field of the Sun is performed, possibly with accuracies better than those attainable in the terrestrial environment. The advantage of using the system of the Lagrange points, in addition to the size of the loop, as said, is that it constitutes a stable frame co-rotating with the Earth, so that the experimental setup is also stable and the measurement may be repeated many times in the same geometrical conditions, averaging out random disturbances. Furthermore, the objects lying in the Ls are in free fall, as is the whole setup, removing kinematical, non-inertial, disturbing or even dominant, effects, typical of terrestrial laboratories. We remark that solar LT field measurements, especially its dynamics, could be an interesting tool to 
address the Sun's interior rotation profile, particularly within the convective zone. Once more the most effective configuration would include $L_{3}$.

\section{Synergy with LISA}

The measurement strategy proposed for $\mathrm{LaDaHaD}$ offers an interesting opportunity for synergy with LISA, a space interferometer designed to reveal gravitational waves (GW) [17]. After the recent detection of a number of signals originating from the merger of pairs of black holes [1] or even pairs of neutron stars [2], the international effort to secure further detections has intensified.

The configuration of LISA is an equilateral triangle $2.5 \times 10^{9} \mathrm{~m}$ in side having freely falling transponders at the end of each side [17]. The centre of the triangle would orbit the Sun along the orbit of the Earth at some $50 \div 65$ million km behind our planet. Laser beams along the arms of LISA would serve both as GW sensors and as controllers of the geometry. It is evident that the closed contour could also be used for Sagnac-like experiments like for LaDaHaD: the LISA triangle would have an area four orders of magnitude smaller than the $\mathrm{LaDaHaD}$ triangle but at the same time it would lead to a seasonal modulation of the inclination angle of the plane of the polygon with respect to the axis of the Milky Way. A collaboration with LISA would be both a precious intermediate step towards the full deployment of LaDaHaD and a relevant autonomous investigation of the GM field of the MW and of the Sun.

\section{Gravitational waves}

Besides the aforementioned possible synergy with LISA, LaDaHaD could also provide an interesting complementary opportunity for the detection of GW. In fact, there is an approach that can employ the same setup and method described for the GM measurement. Recently there has been a proposal to exploit (in a circum-terrestrial environment) a Sagnac-like measurement along a triangular loop in space, for detecting GW originated from intermediate mass black hole mergers [32]. Already in the 1990s, Sun, Fejer, Gustafson and Byer [45], starting from a previous idea of Weiss, proposed and analyzed a so called zero-area Sagnac interferometer for the detection of GW. The zero-area Sagnac interferometer organizes the path of light beams so that the contoured area is zero. In practice, one has a pair of twisted half loops and each beam travels along the two loops once clockwise, and once counter-clockwise. The consequence is that the signal of the kinematic and of the effective rotations cancel; if, however, the strain induced by a GW is present, the transient difference in the length of the arms of the interferometer maintains its effect and is recorded as a residual asymmetry in the phases of the beams. On Earth the principle works, but the storage time in one arm of the interferometer can hardly be longer than a ms. In the case of our Lagrangian system a zero-area configuration could be achieved including Earthbased stations and using for instance an $L_{4}-L_{2}-$ Earth $-L_{5}-L_{2}-$ Earth $-L_{4}$ circuit. In that case the "storage time" (here the flight time) in a half loop would be of the order of one thousand seconds. The angle between the arms (important in order 
for the strain of the $\mathrm{GW}$ to be manifested) would be $120^{\circ}$ rather than $90^{\circ}$, but the permanence time would be $10^{6}$ times longer than in a terrestrial device.

\section{Relativistic positioning}

Together with the direct scientific return provided by LaDaHaD in demonstrating GR effects at the scale of the Solar System, the whole concept and the same principle could provide important support to any other mission in space outside the Earth's environment. One of the delicate problems concerning all such missions is the localization and guidance of the spacecraft. Such a task is currently performed by a combination of different techniques, including self-guidance using the image of the sky. Most often, however, and for most of the time, the mission control is carried out from Earth. There, ranging methods give good results, however the transverse positioning progressively worsens while the distance increases. The situation could be much better with beacons located in the Sun-Earth Lagrange points, like in the configuration proposed for testing the GR effects. In fact, very little additional equipment would be needed beyond what is already required by the main experiment: each spacecraft of the LaDaHaD system should act as a stable emitter either of a continuous electromagnetic wave at a fixed frequency or of pulses issued at a fixed rate. The emission should be isotropic or at least over wide angles. The positioning method based on local time measurements autonomously performed by the user (the object to be positioned) is intrinsically relativistic (we refer to it as a Relativistic Positioning System: RPS) and has been proposed and discussed in various papers, and in particular in [47].

In short, the idea is as follows. We start from a physical basis composed of (at least) four independent emitters of electromagnetic pulses (or continuous harmonic waves) whose emission rate is as stable as possible, and is known. The emitters form a physical frame with respect to which the positioning is made. In four dimensions the wave-fronts of the pulses from the sources cover space-time with a regular array made of "cages" whose edges are parallel to the propagation directions from the emitters and whose size (when splitting space and time) is the proper wavelength (or distance from successive pulses) and the proper emission period, i.e. the wavelength and period measured in the reference frame of the emitter. In such a four-dimensional grid, a traveler is represented by a continuous line (its world-line) crossing the boundaries of successive "cages" (see Fig. 3, drawn in two, i.e. $1+1$, dimensions); the situation is described and visually depicted in [48]. While receiving the signals from the various sources, the traveler must have the possibility to distinguish and identify each source, then the traveler counts the pulses and measures on their own clock the time intervals between the arrivals of one pulse and the next. If the signals are continuous, equivalently, the traveler takes note of the relative phase between the incoming waves. The measured time spans are proportional to the intervals (in the GR sense) between successive arrival events. Simple geometrical considerations allow the user to reconstruct, from the measured sequences of arrival times, their own coordinates along the light cones, then, more familiarly, in the reference frame of the emitters. 


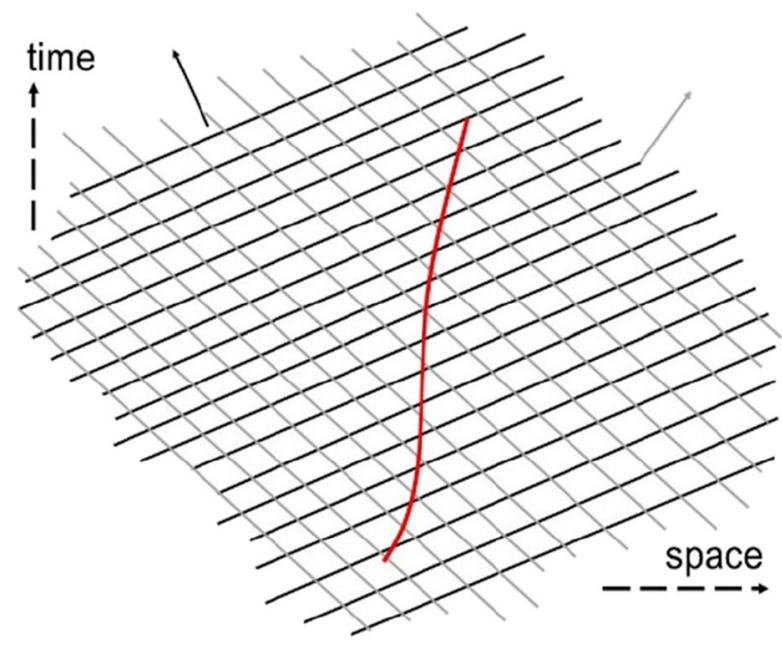

Fig. 3 Bidimensional (space+time) view of the space-time grid formed by the wave fronts from the emitters. The red line is the world-line of a travelling user

Such an RPS has been conceived and tested using pulsars as beacons [40]. In the case of $\mathrm{LaDaHaD}$, the emitters in the L points would act as "artificial pulsars". Once more, the advantage of the Lagrange points is that they form a reference frame co-moving with the Earth. Furthermore, the size of the base is such that geometric dilution effects can be minimized.

\section{Relativistic modeling of the solar system}

The advancement in astronomical observations and instrumentation requires reconstructing light propagation at a very high level of precision, whenever the accuracy of the measurements, in terms of lengths, is comparable to the square root of the inverse curvature of the background geometry due to the distribution of the sources of gravity. This is particularly needed for space missions such as LaDaHaD and Gaia, which necessarily require extremely accurate astrometric observations modeled within a fully, suitably accurate, relativistic framework.

The new model for 'tracing' photons developed for the Gaia mission (see $[15,16]$ and references therein; [54]) is now part of the European excellence in the field of modeling photon paths in low gravity and of the Gaia effort of building, with two independent methodologies, the first fully relativistic reconstruction, both differential and absolute, of the celestial sphere. The other approach, GREM, is described in [29].

In particular, RAMOD (Relativistic Astrometric MODel) [5, 14, 18, 19] naturally recovers the seminal and fundamental works of Kopeikin and Schäfer [30] and Klioner ([29] and references therein) and provides a fully general-relativistic analysis of the inverse ray-tracing problem based upon a measurement protocol in GR [20]. The latter uses a $3+1$ characterization of space-time in order to measure 
physical phenomena along the proper time and on the rest-space of a set of fiducial observers. Then, the unknown of RAMOD is the four-dimensional local line-of-sight as measured by the fiducial observer at the point of observation.

While the physical spatial component is used for the Gaia data modeling and compared with the coordinated one used in GREM, the time component can be exploited for the LaDaHaD measurements. Moreover, the RAMOD method is close [3] to the Time Transfer Function formalism (TTF, [34, 52]) that stands as a development of Synge's World Function, an integral approach based on the principle of minimal action. While the World Function is an implicit equation of the photon trajectory that is nearly impossible to solve, the TTF formalism gives up some generality to provide important information about the propagation of an electromagnetic wave between two points at finite distance from one another.

This scientific activity has not only contributed to establishing novel theoretical and numerical methods for treating light propagation consistently with the covariant approach of GR, but has also provided new strategies to go further in testing gravity and its connection with the scientific potential of a local measurement entangled in the varying gravitational fields of the Solar System. Any inconsistency in the relativistic model(s) would invalidate the quality and reliability of the estimates, indeed all the relevant scientific outputs, and, in addition, could be the clue for uncovering new effects. In this regard, $\mathrm{LaDaHaD}$ can offer the unique opportunity to complete the theoretical comparison of the existing approaches and set the scientific case for further developments and applications, even in the quantum gravity regime [28].

\section{More opportunities}

Pulsar timing. The use of the emitters as "artificial pulsars" located at well-defined positions in space lends an opportunity also for astrophysicists. In fact, the artificial pulsars may be used to help timing the natural ones.

Gravitational potential mapping at the $L$ points Studying the behavior of the spacecraft in each $\mathrm{L}$ point can be a method to map the corresponding gravitational potential. The movements of a station around its own L point can indeed be reconstructed using the other stations as a reference frame and applying the RPS. The reconstructed details of the movement give a map of the local effective potential in the vicinity of the $\mathrm{L}$ point.

Recognition of the space environment of $L_{4}$ and $L_{5} \quad L_{4}$ and $L_{5}$ are the most distant Lagrange points (not considering $L_{3}$ ), so until now not much attention has been paid to their environment, even though it would probably be quite interesting. ESA is indeed planning to send a mission to $L_{5}$ [22] that could be combined with LaDaHaD. $L_{4}$ and $L_{5}$ are (weakly) stable, being local (shallow) minima of the effective potential (including Coriolis forces). This feature favors positioning the transponders and stations of $\mathrm{LaDaHaD}$ there; at the same time, the local potential well may imply the presence of celestial bodies trapped there. It is already known that at least one asteroid (2010 TK7) is dwelling in $L_{4}$ [13]: it is the first "Trojan asteroid" of the Earth 
to be discovered. More could be there and in $L_{5}$ too. Missions sent to lay transponders/beacons of $\mathrm{LaDaHaD}$ in $L_{4}$ and $L_{5}$ would offer the possibility to explore the space around those points.

\section{Scientific and technological requirements}

In order to verify the practical possibility to carry the present proposal to completion, a number of detailed studies both on the scientific and on the technological aspects of the related missions are necessary. On the side of scientific and conceptual tools a list of requirements follows.

a) The Solar System is of course not made of the Sun-Earth pair only. An analysis of the gravitational perturbations induced by the other celestial bodies is required, both to understand the stability of the positions of the Lagrange points and to assess the influence on the signal that would be measured.

b) All effects have to be analyzed from the view point of GR in order to build a consistent interpretation frame for the data in view of the expected results.

c) A detailed study of the effects of deviations from GR in the framework of PPN formalism is in order. An analysis is already present in [6] and the relevant parameters taken into account there have been $\alpha_{1}$ (accounting for the presence and relevance of preferred reference frames) and $\gamma$ (accounting for the effect of spatial curvature alone). The possible relevance of these and other parameters must be discussed at the scale of the inner Solar System. Furthermore, the possible presence of galactic or even relic, GM fields must be considered and modeled in order to provide appropriate templates for the interpretation of the results.

d) Since the position at the L points is crucial, it is of paramount importance to study the orbits of the spacecraft of the experiment around those points and the related dynamics and stability. Libration movements are expected in $L_{4}$ and $L_{5}$, where the orbits can be stable, whereas weakly unstable Lissajous orbits are typical of $L_{1}$ and $L_{2}$ (saddle points of the effective potential). Stabilization strategies must be envisaged. One should also account for the proper motion of the L's with respect to the Earth, due to the eccentricity of the orbit of our planet.

e) A model must be developed accounting for the effects of the movements of the stations, according to point d), on the final evaluation of the time of flight difference along closed paths among the L points. Semi-analytical investigations of the main periodic orbits around the Lagrange points $[8,9,37]$ provide accurate approximations to model distance variations. They can also be used as seeds for numerical surveys of the unstable dynamics around $L_{1}$ and $L_{2}$.

Coming to technological needs, many aspects require in depth analyses and improvements with respect to their present implementations. 
a) The core measurement of $\mathrm{LaDaHaD}$ concerns time. As stated in previous sections, the interval between the arrivals of the clockwise and counterclockwise pulses is of the order of $10^{-13} \mathrm{~s}$ (in the best case $10^{-12} \mathrm{~s}$ ). In order to attain a $1 \%$ accuracy, the clock (and the measurement process) must have a sensitivity of at least $10^{-15} \mathrm{~s}$. This is feasible in terrestrial laboratories, where one can achieve results one or two orders of magnitude better than that. One has, however, to think of atomic clocks in space, onboard an observatory located in one of the $\mathrm{L}$ points (such as $L_{1}$ or $L_{2}$ ), endowed with the required accuracy and a stability sufficient for a long mission (not less than months). A possible solution in order to ensure the best accuracy and for the longest time would be to permanently and for all configurations (see the section on deployment strategy) keep an Earth laboratory as a hub of our experimental polygonal network. In any case, the need to have and control clocks and oscillators in space cannot be dispensed of completely, so that the problem must be carefully studied.

b) The main station must be equipped also with an appropriate computational capacity although the requirement there is not very stringent: the algorithm to be used, in the case of RPS, is linear. More important is the onboard data storage capacity, connected with an effective communication channel for transferring information to the Earth.

c) The technology of the transponders to be used at the other (with respect to the main one) stations has to be analyzed carefully. Each transponder is expected to work as much as possible like a mirror, with a fixed and as short as possible delay between the arrival and the bounce back of the incoming pulses. The same problem has to be tackled for the phase-locked transponders of LISA.

d) In case $\mathrm{LaDaHaD}$ is used as a base for positioning and navigation in the Solar System, each L point must host an emitter of regular pulses, possibly combined with the transponder. Each emitter needs be controlled by an atomic clock; the main constraints are not on the frequency $(\mathrm{GHz}$ or even $\mathrm{MHz}$ repetition times for the pulses are sufficient), but rather on the stability over months or even years: if the RPS has to be considered as permanent equipment for navigation.

e) Special attention must be paid to the design of all antennas installed onboard.

f) All electronic equipment, at all stations, of course needs an appropriate power supply, whose main components are solar cells and storage capacity.

\section{Deployment and measurement strategy}

The core of the $\mathrm{LaDaHaD}$ experiment is the local time measurement of time of flight differences along closed paths in space, based on the Sun-Earth Lagrange points. The full deployment of the system is quite burdensome and will require a long time to be completed. It is however possible to define a strategy relying on progressive steps, allowing data collection from the beginning. As previously mentioned, the $L_{2}$ 
and $L_{1}$ points already host various space missions. In particular, in $L_{2}$ we find Gaia, which is equipped for communicating with the Earth and could technically perform tasks much like the ones demanded of the proper stations of $\mathrm{LaDaHaD}$. The planned duration of the mission is close to the end, but, most likely, the spacecraft can live longer than the official duration of the mission, especially if the assignments are simplified and reduced to act as a bounce back station for $\mathrm{LaDaHaD}$; it is unlikely that the life of the spacecraft may be prolonged for many years, but anyway we could in principle consider its presence also. Furthermore, the Earth as such may be included as a vertex of the space polygons we want to use; of course in the case of ground-based stations one has to include the effect of the diurnal rotation of the planet, which implies a discontinuous operation and the need to account for a peculiar relative motion.

Relying on the cited pair of bases, a first step (not a trivial one, in any case) could consist of sending a mission to $L_{5}$ (or $L_{4}$ ), then the triangle Earth $-L_{2}-L_{5}$ would be available for data taking. An advantage of having a laboratory on the ground would be the possibility to use the best atomic clocks, whose accuracy would in principle permit the detection of solar GM terms at levels better than 1 part in $10^{3}$. Of course, the perturbations induced by the atmosphere should be carefully analyzed, together with the presence, close to the ground station, of the terrestrial GM field.

The next step could be to place one additional spacecraft in $L_{1}$. A new triangle would then be at hand, giving a useful degree of redundancy. The whole system would contain two possible intertwined loops, in the shape of the zero-area Sagnac interferometer mentioned in the section of gravitational waves. That particular configuration has also been called "butterfly wings"; in this case the "wings" would be in almost closed position and the time of flight along each loop (each "wing") would be $\sim 600 \mathrm{~s}$, with an angle between the long and the short arm equal to $60^{\circ}$. An improvement of this configuration would be achieved replacing Gaia in $L_{2}$ with a new dedicated station.

One further improvement would be realized by sending a station to $L_{4}$. Now the possible loops would be seven (including the Earth) offering redundancy and alternative strategies. For GW monitoring, the wings of the butterfly would now be open and symmetric with respect to the Sun-Earth axis rather than the Earth $-L_{5}$ direction.

The complete network of stations would be attained sending a spacecraft also in $L_{3}$. This would represent the most sensitive configuration for the measurement of the solar gravito-magnetism and for the study of the internal dynamics of the Sun. The highest sensitivity for deviations from GR would also be attained.

Funding Open access funding provided by Politecnico di Torino within the CRUI-CARE Agreement.

Open Access This article is licensed under a Creative Commons Attribution 4.0 International License, which permits use, sharing, adaptation, distribution and reproduction in any medium or format, as long as you give appropriate credit to the original author(s) and the source, provide a link to the Creative Commons licence, and indicate if changes were made. The images or other third party material in this article are included in the article's Creative Commons licence, unless indicated otherwise in a credit line to the material. If material is not included in the article's Creative Commons licence and your intended use is not permitted by statutory regulation or exceeds the permitted use, you will need to obtain permission directly from the copyright holder. To view a copy of this licence, visit http://creativecommonshorg/licenses/by/4. $0 /$. 


\section{References}

1. Abbott, B.P., et al.: Observation of gravitational waves from a binary black hole merger. Physic. Rev. Lett. 116, 061102-1, 061102-16 (2016)

2. Abbott, B. et al.: GW170817: Observation of gravitational waves from a binary neutron star inspiral. Phys. Rev. Lett. 119, 161101 (2017)

3. Bertone, S. et al.: Time transfer functions as a way to validate light propagation solutions for space astrometry. Classic. Quantum Grav. 31, 015021 (2014)

4. Beverini, N. et al.: Toward the perfect square ring laser gyroscope. Photonic technologies, 2014 Fotonica AEIT Italian Conference 1-4 (2014)

5. Bini, D., Crosta, M., de Felice, F.: Orbiting frames and satellite attitudes in relativistic astrometry. Classic. Quantum Grav. 20, 4695-4706 (2003)

6. Bosi, F., et al.: Measuring gravitomagnetic effects by a multi-ring-laser gyroscope. Physic. Rev. D 84, 122002-1, 122002-23 (2011)

7. Carignan, C., Chemin, L., Huchtmeier, W.K., Lockman, F.J.: The extended HI rotation curve and mass distribution of m31. Astrophys. J. 641(2), L109-L112 (2006)

8. Ceccaroni, M., Celletti, A., Pucacco, G.: Halo orbits around the collinear points of the restricted three-body problem. Physica D 28-42, 317 (2016)

9. Celletti, A., Pucacco, G., Stella, D.: Lissajous and Halo orbits in the restricted three-body problem. J. Nonlinear Sci. 25, 343-370 (2015)

10. Ciufolini, I., Pavlis, E.: A confirmation of the general relativistic prediction of the LenseThirring. Nature 431, 958-960 (2004)

11. Ciufolini, I., et al.: Testing gravitational physics with satellite laser ranging. Europ. Physic. J. Plus 126(8), 72 (2011)

12. Ciufolini, I., et al.: A test of general relativity using the LARES and LAGEOS satellites and a GRACE Earth gravity model. Europ. Physic. J. C 76, 120 (2016)

13. Connors, M., Wieger, P., Veillet, C.: Earth's Trojan asteroid. Nature 475(7357), 481-483 (2011). https://doi.org/10.1038/nature 10233

14. Crosta, M., Vecchiato, A.: Gaia relativistic astrometric models. I. Proper stellar direction and aberration. Astron. Astrophys. 509, A37 (2010)

15. Crosta, M.: Tracing light propagation to the intrinsic accuracy of spacetime geometry. Classic. Quantum Gravit. 28, 235013 (2011)

16. Crosta, M., et al.: The ray tracing analytical solution within thr RAMOD framework. The case of a Gaia-like observer. Classic. Quantum Gravit. 32, 165008 (2015)

17. Danzmann, K., et al.: A proposal in response to the ESA call for $l_{3}$ mission concepts. LISA Laser Interferometer Space Antenna (2017)

18. De Felice, F. et al.: A general relativistic model of light propagation in the gravitational field of the solar system: the static case. Astrophysic. J. 607, 580-595 (2004)

19. De Felice, F. et al.: A general relativistic model of light propagation in the gravitational field of the solar system: the dynamical case. Astrophysic. J. 653, 1552-1565 (2006)

20. De Felice, F., Bini, D.: Classical Measurements in Curved Space-Times. Cambridge, Cambridge University Press (2010)

21. Di Virgilio, A., et al.: A ring lasers array for fundamental physics. C. R. Physique 15, 866-874 (2014)

22. ESA: European Space Agency: Future Lagrange Mission (2017)

23. Everitt, C., et al.: Gravity probe B: Final results of a space experiment to test general relativity. Physic. Rev. Lett. 106, 221101-1, 221101-5 (2011)

24. Freese, K.: Status of dark matter in the universe. arXiv:1701.01840 [astro-ph.CO], 1-32 (2017)

25. Gerhard, O.: Mass distribution in our Galaxy. Space Sci. Rev. 100(1), 129-138 (2002)

26. Hodges-Kluck, E., Miller, M., Bregman, J.: The rotation of the hot gas around the milky way. Astrophys. J. 822(1), 1-11 (2016)

27. Honma, M. et al.: Fundamental parameters of the milky way galaxy based on VLBI astrometry. Publ. Astron. Soc. Japan 64(136), 1-13 (2012)

28. Khavkine, I.: Quantum astrometric observables: Time delay in classical and quantum gravity. Physic. Rev. D 85(12), 124014 (2012)

29. Klioner, S.A.: A practical relativistic model for microarcsecond astrometry in space. Astronomic. J. 125, 1580-1597 (2003) 
30. Kopeikin, S.M., Schäfer, G.: Lorentz covariant theory of light propagation in gravitational fields of arbitrary moving bodies. Physic. Rev. D 60, 124002 (1999)

31. Kramer, M. et al.: Strong-field tests of gravity with the double pulsar. Ann. Phy 15(1-2), 34-42 (2006)

32. Lacour, S., et al.: SAGE: finding IMBH in the black hole desert. Classic. Quantum Gravit. 36(19), 1-33 (2019). arXiv:1811.04743v1 [gr-qc] (2018)

33. Lense, J., Thirring, H.: ÜBer den Einfluss der Eigenrotation der zentralkörper auf die Bewegung der Planeten und Monde nach der Einsteinschen Gravitationstheorie. Physikalische Zeitschrift 19, 156-63 (1918)

34. Le Poncin-Lafitte, C., Teyssandier, P.: Influence of mass multipole moments on the deflection of a light ray by an isolated axisymmetric body. Physic. Rev. D 77, 044029 (2008)

35. Lucchesi, D.M. et al.: General relativity measurements in the field of earth with Laser-Ranged satellites: state of the art and perspectives. Universe 5, 1-32 (2019)

36. Massey, R., Kitching, T., Richard, J.: The dark matter of gravitational lensing. Rep. Prog. Phys. 73(8), 86901-86949 (2010)

37. Páez, R., Locatelli, U., Efthymiopoulos, C.: New Hamiltonian expansions adapted to the Trojan problem. Celestial Mechan. Dynamic. Astron. 126(4), 519-541 (2016)

38. Planck Collaboration: Planck 2018 results. I. Overview and the cosmological legacy of Planck. arXiv:1807.06205v2 [astro-ph.CO], 1-62 (2019)

39. Ruggiero, M., Tartaglia, A.: Gravitomagnetic effects. Nuovo Cimento B 117, 743-768 (2002)

40. Ruggiero, M.L., Capolongo, E., Tartaglia, A.: Pulsars as celestial beacons to detect the motion of the earth. Int. J. Modern Physic. D 20(6), 1025-1038 (2011)

41. Sagnac, M.G.: The demonstration of the luminiferous aether by an interferometer in uniform rotation. Comptes Rendus de 1'Acadé,mie des Sciences 157, 708-710 (1913)

42. Saxton, C.J.: Galaxy stability within a self-interacting dark matter halo. Mon. Not. R. Astron. Soc. 430(3), 1578-1598 (2013)

43. Sofue, Y., Rubin, V.: Rotation curves of spiral galaxies. Annu. Rev. Astron. Astrophys. 39, 137-174 (2001)

44. Sukanta, B., Grishchuk, L.P.: Observational determination of squeezing in relic gravitational waves and primordial density perturbations. Phys. Rev. D 66, 043529 (2002)

45. Sun, K.-S., Fejer, M.M., Gustafson, E., Byer, R.L.: Sagnac Interferometer for Gravitational-Wave Detection. Physic. Rev. Lett. 76(17), 3053-3056 (1996)

46. Tahir, N., De Paolis, F., Qadir, A., Nucita, A.: Seeing the halo rotation of nearby spiral galaxies using Planck data. Arabian J. Math. 8, 193-199 (2019)

47. Tartaglia, A., Ruggiero, M.L., Capolongo, E.: A null frame for spacetime positioning by means of pulsating sources. Adv. Space Res. 47, 645-653 (2011)

48. Tartaglia, A.: Relativistic space-time positioning: principles and strategies. Acta Futura n.(7), 111124. https://doi.org/10.2420/AF07.2013.111 (2013)

49. Tartaglia, A., Di Virgilio, A., Belfi, J., Beverini, N., Ruggiero, M.: Testing general relativity by means of ring lasers. Eur. Phys. J. Plus 132(73), 1-10 (2017)

50. Tartaglia, A. et al.: How to use the Sun-Earth Lagrange points for fundamental physics and navigation. Gen. Relativ. Gravit. 50(1), 1-21 (2018)

51. Tartaglia, A.: Detecting the angular momentum of the galactic dark halo. Adv. Space Res. 64(2), 545-550 (2019)

52. Teyssandier, P., Le Poncin-Lafitte, C.: General post-Minkowskian expansion of time transfer functions. Classic. Quantum Gravit. 25, 145020 (2008)

53. Thirring, H.: ÜBer die Wirkung rotierender ferner Massen in der Einsteinschen Gravitationstheorie. Physikalische Zeitschrift 19, 33-39 (1918)

54. Vecchiato, A., et al.: The global sphere reconstruction for the Gaia mission in the astrometric verification unit. In: Proceedings: Software and Cyberinfrastructure for Astronomy II, vol. 8451, p. 84513C (2012). Society of Photo-Optical Instrumentation Engineers (SPIE). https://doi.org/10.1117/12.926098

55. Weisberg, J.M., Huang, Y.: Relativistic Measurements from Timing the Binary Pulsar PSR B1913+16. Astrophysic. J. 722(2), 1-10 (2016)

Publisher's note Springer Nature remains neutral with regard to jurisdictional claims in published maps and institutional affiliations. 